Draft VERsion November 8, 2018

Preprint typeset using $\mathrm{LAT}_{\mathrm{E}} \mathrm{X}$ style emulateapj v. 5/2/11

\title{
STAR STREAMS AND THE ASSEMBLY HISTORY OF THE GALAXY
}

\author{
Raymond G. CARlberG \\ Department of Astronomy \& Astrophysics, University of Toronto, Toronto, ON M5S 3H4, Canada \\ Draft version November 8, 2018
}

\begin{abstract}
Thin halo star streams originate from the evaporation of globular clusters and therefore provide information about the early epoch globular cluster population. The observed tidal tails from halo globular clusters in the Milky Way are much more shorter than expected from a star cluster orbiting for 10 Gyr. The discrepancy is likely the result of the assumption that the clusters have been orbiting in a non-evolving galactic halo for a Hubble time. As a first step towards more realistic stream histories, a toy model that combines an idealized merger model with a simplified model of the internal collisional relaxation of individual star clusters is developed. On the average, the velocity dispersion increases with distance causing the density of the stream to decline with distance. Consequently, the streams visible in current data will normally be some fraction of the entire stream. Nevertheless, the high surface density segment of the stellar streams created from the evaporation of the more massive globular clusters should all be visible in low obscuration parts of the sky if closer than about $30 \mathrm{kpc}$. The Pan-STARRS1 halo volume is used to compare the numbers of halo streams and globular clusters.

Subject headings: dark matter; Local Group; galaxies: dwarf; globular clusters; Galaxy: halo
\end{abstract}

\section{INTRODUCTION}

The current globular cluster population is the remnant of a larger population which lost its lower mass members to evaporation driven by internal two-body relaxation and tides (Fall \& Rees 1977; Gnedin \& Ostriker 1997; Fall \& Zhang 2001). However, at least some fraction of those remnants are visible as the thin stellar streams found in the halo (Grillmair \& Carlin 2016). Combining globular clusters and their remnant stellar streams into one dynamical picture offers the possibility of providing additional, less "survivor" biased, insights into the early epoch globular cluster population and the assembly history of the Milky Way halo.

Two-body relaxation in globular clusters causes stars outside the core to gain energy, which drives a gradual evaporation of the cluster in the tidal field of the galaxy. The evaporating stars form tidal star streams that are useful for measuring the shape of the galactic potential (Binney 2008, Law \& Majewski 2010; Eyre \& Binney 2011; Sanders \& Binney 2013; Bovy et al. 2016) and detecting the many dark sub-halos that are expected to be present (Klypin et al. 1999, Moore et al. 1999). The dynamical analysis of stream formation usually starts with star clusters instantaneously inserted into a fixed background potential, although that potential may contain a small fraction of its mass in the form of orbiting sub-halos. Some simulations use an approximation that prescribes the release of stars near the tidal surface (Küpper et al. 2012). In n-body simulations of stream formation particle softening makes the clusters collisionless, although tidal heating in a reasonably elliptical orbit is sufficient to cause mass loss. Although the mass loss rate varies around the orbit, it is periodic so that the the mean mass loss rate is constant and the dynamical properties of the stars along the stream repeat nearly exactly along the stream (?Carlberg 2015a). Much valuable insight results from the analysis of streams and sub-halos

carlberg@astro.utoronto.ca orbiting in a non-evolving potential (Küpper et al. 2012 Carlberg 2015a; Bovy et al. 2017) but it does overlook the fact that the overall halo potential and is assembled over comparable time-scales.

Observed stellar streams, or stream segments, are typically about $10 \mathrm{kpc}$ such as the well-studied Pal 5 (Odenkirchen et al. 2001; Grillmair \& Dionatos 2006b) and GD-1 (Grillmair \& Dionatos 2006a) streams. There length is somewhat puzzling because globular clusters are 10 Gyr old systems, that release stars into the tidal stream at typical velocities, about $5 \mathrm{~km} \mathrm{~s}^{-1}$, which should lead to long streams, $\sim 50 \mathrm{kpc}$. How the process of assembly of the globular clusters into our galactic halo affects the properties of the stellar streams, and, conversely, how stellar streams might provide insight into the history of the halo and its globular cluster population is the goal of this paper.

The internal dynamical evolution of globular star clusters in a tidal field is fairly well understood (Spitzer 1987 Davies 2013). The mass evolution of globular clusters in the galaxy halo has been discussed at length (Fall \& Rees 1977, 1985, Gnedin \& Ostriker 1997; Fall \& Zhang 2001; Gieles et al. 2011; Lamers et al. 2013). Renaud et al. (2011) have developed an approach to the problem of a star cluster evaporating in a merging system, concentrating on the star cluster evolution. The rate of evaporation depends on the internal two body relation time and the local tidal field. The tidal field changes from the site of cluster formation to its accretion into the galactic halo. As a cluster evaporates the rate and velocity at which stars are ejected into the tidal streams changes, which in turn affects the density of the tidal stream created.

This paper explores how the observed star streams and remnant halo globular clusters are related with their history of assembly into the galaxy. A toy model of accretion infall of a satellite galaxy containing model globular clusters is developed. The satellite is represented as an evolving analytic potential which falls into the host with dynamical friction. The particles in the model clusters 
are subjected to a simple Monte Carlo heating procedure which mimics the relaxation processes of globular clusters with the expected mass and size scaling. The evolution of the clusters with time and the resulting properties of the streams on the sky are presented. As an initial practical application, the number of star streams in the recent Pan-STARRS1 volume is compared to the number of globular clusters.

\section{A SIMPLIFIED N-BODY MODEL}

An n-body simulation of the dynamical evolution of a star cluster starting in a satellite galaxy which merges with its host ideally requires a large numbers of particles and a high precision n-body code that follows stellar two-body interactions but has no two-body interactions with dark matter particles. Here the problem is split into two parts, one being to model the dark matter merger of a satellite and host galaxy and the other to provide internal evolution of the star cluster. The aspect of galaxy merging needed here is a satellite galaxy potential that loses orbital energy and blends into the host galaxy releasing its globular clusters to orbit in the host galaxy potential. Analytic potentials for the host and satellite combined with dynamical friction can meet these requirements. There is no need in this exploratory study to have a full dynamical model of the merger process or detailed cosmological starting conditions.

The the initial host galaxy model is Milky Way 2014 of Bovy (2015). The halo mass component of the model potential absorbs mass from the satellite as it merges, but there is no violent relaxation. A population of dark matter sub-halos is included in the host galaxy as fixed mass Hernquist spheres following the prescriptions of Carlberg \& Grillmair (2016). The sub-halos extend out to 360 kpc. Sub-halos below a mass of $0.9 \times 10^{7} \mathrm{M}_{\odot}$ are not included because they make essentially no difference to the streams studied here.

\subsection{The Satellite Galaxy Starting Conditions}

The infalling galaxy needs to have a well-defined mass to allow the dynamical friction formula to be used. The Hernquist model (Hernquist 1990), $\phi_{H}\left(r, a_{s}\right)=$ $-G M_{s} /\left(r+a_{s}\right)$ is a convenient choice where $M_{s}$ and $a_{s}$ are the satellite mass and scale radius, respectively. The Bovy MW2014 potential has a halo mass inside the 245 $\mathrm{kpc}$ virial radius of $8.1 \times 10^{11} \mathrm{M}_{\odot}$ or 8.992 dimensionless mass units, where the mass unit is $9.006 \times 10^{10} \mathrm{M}_{\odot}$. The mass lost is added to the host galaxy halo NFW potential (Navarro et al. 1997) which has a scale radius of 2 dimensionless units in MW2014. The satellite needs to have a similar dark matter density, so $a_{s}=2\left(M_{s} / 8\right)^{1 / 3}$. The baseline satellite mass, $M_{s}$, is chosen to be 2 mass units with $a_{s}=1.26$ which can be considered as a satellite with $22 \%$ of the mass of the host halo within the virial radius.

Ideally, the satellite galaxy orbit would be drawn from a cosmological simulation, but with a single infalling galaxy all that is required is a reasonably representative starting point someplace near the virial radius. The satellite galaxy is started in a bound orbit, at a galactocentric $x$ of $240 \mathrm{kpc}$ and above the plane of the MW2014 model at $z$ of $120 \mathrm{kpc}$. The satellite is given an initial tangential velocity equal to 0.35 of the local circular ve-

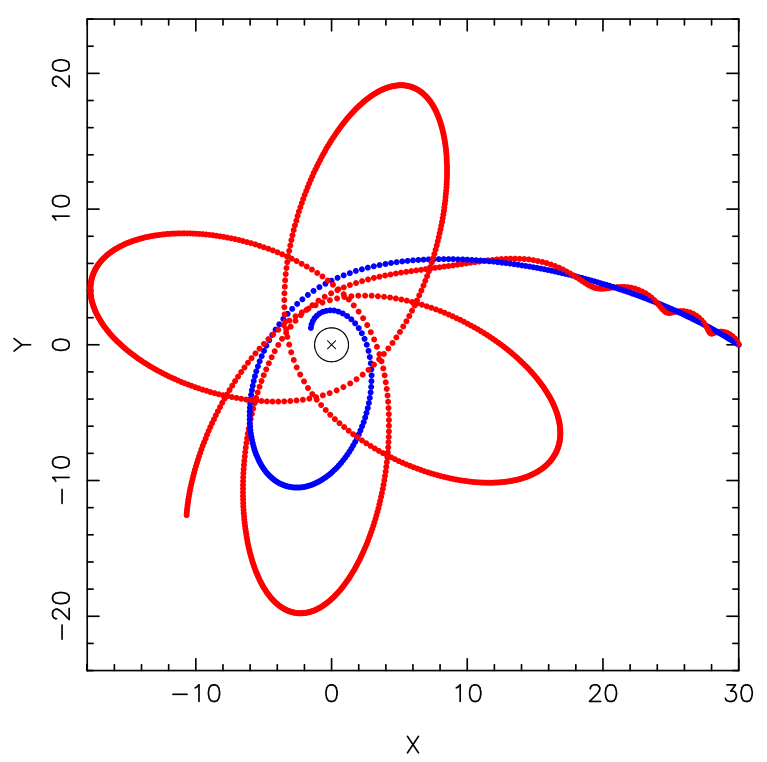

FIG. 1. - The blue points show the orbit of the center of the satellite potential with $M_{s}=0.3$. The red points show the orbit of the center of a single model star cluster. The scale is in the dimensionless units of $8 \mathrm{kpc}$ per unit. More massive satellites sink more quickly with $M_{s}=2$ having only a quarter turn orbit.

locity and an inward radial velocity of 0.61 of the local circular velocity.

\subsection{The Merger Model}

An accurate violent relaxation model is not essential for this investigation which focuses on the star clusters and their tidal streams. Two analytic time-varying analytic potentials moving together with dynamical friction give an approximate description of the merger. Chandrasekhar's formula (Binney \& Tremaine 2008, BoylanKolchin et al. 2008) is used to evaluate the dynamical friction, with $\ln \Lambda=10$ adopted. The NFW dark matter halo in the MW2014 potential is used to calculate the local mass density for friction. The NFW is given a small core radius equal to the MW2014 bulge core radius to avoid the singularity when the satellite is dragged to the center of the host galaxy. As the satellite merges into the host, it loses mass and expands to spread over the host halo potential. The key dynamical quantity is $\Omega_{s}$, the local circular frequency of the satellite orbit, which is estimated from the local acceleration in the host galaxy, $\Omega_{s}=\sqrt{\mathbf{a} \cdot \mathbf{r}} / r$. The spread in circular frequency of the orbit at radius $r$ across the scale radius of the satellite, $a_{s}$, is approximated $\delta \Omega_{s} \simeq \Omega_{s} a_{s} / r$. Therefore, the rate of satellite mass decrease due to orbital divergence is modeled as,

$$
\frac{d M_{s}}{d t}=-\frac{M_{s} a_{s}}{r^{2}} \sqrt{\mathbf{a} \cdot \mathbf{r}}
$$

with the lost mass being added to the host halo. The scale radius of the satellite, $a_{s}$, expands as

$$
\frac{d a_{s}}{d t}=\frac{a_{s}}{r} \sqrt{\mathbf{a} \cdot \mathbf{r}}
$$

These ideas are implemented in the simulation, with the maximum scale radius of the satellite limited to 5 times the scale radius of the NFW of the host galaxy. The mass 


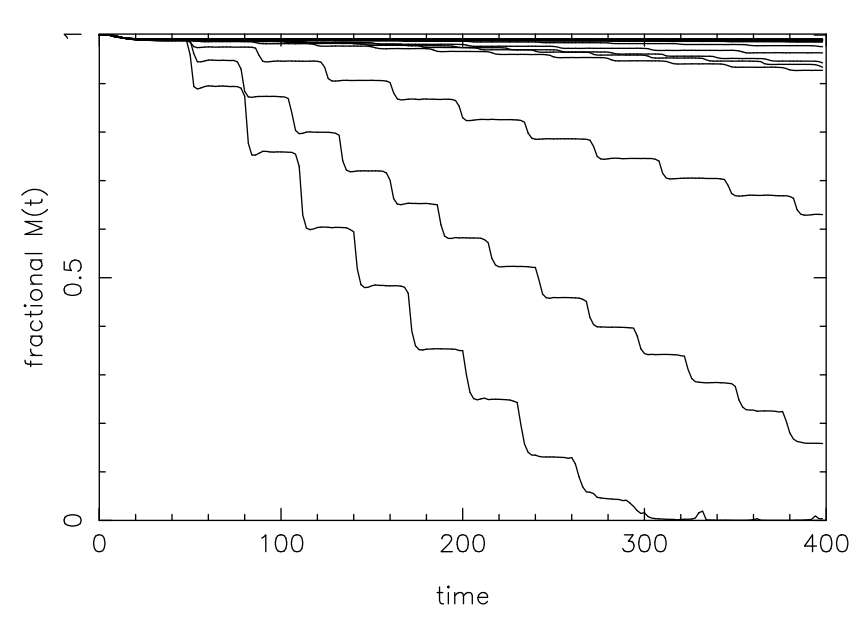

FIG. 2.- Mass as function of time for the 24 clusters each with mass $0.9 \times 10^{4} \mathrm{M}_{\odot}$ with only tidal heating. The internal relaxation model is turned off for the purpose of comparison.

taken from the satellite is added to the host galaxy halo without changing the scale radius. Under this process the satellite orbits into the center of the host galaxy, expands and its mass goes to zero. An example merger orbit is shown in Figure 1.

\subsection{Initial Conditions and Gravity Code}

Star formation occurs in galactic gas disks, which includes the dense star systems that are the likely current epoch equivalents of globular cluster progenitors. Observationally, the formation of globular clusters may be enhanced in vigorously star forming systems associated with interacting disk galaxies (Whitmore et al. 1999). The numerical modeling of the formation of progenitors to globular clusters (Kravtsov \& Gnedin 2005; Bournaud et al. 2008; Portegies Zwart et al. 2010 Renaud et al. 2016 ) is being developed. The simulations find a steep cluster mass distribution, similar to that observed in the dense stellar systems, nearly $M^{-2}$. The simulations here follow a single cluster mass at a time, but simulations at different masses can be combined with appropriate weights to determine how a mass distribution will evolve.

The star clusters start as King models with a concentration parameter of $W_{0}=4$. There are 50,000 particles in a single star cluster. A model cluster of mass $M_{c}$ at an orbital radius $r_{o}$ in the satellite is given an outer radius scaled to the local tidal radius, $r_{t}=\left(M_{c} / 3 M_{t}\right)^{1 / 3} r_{o}$, with the tidal mass $G M_{t}=r \mathbf{a} \cdot \mathbf{r}$. The acceleration is calculated from the satellite galaxy potential alone. The cluster particles are evolved with a fast parallel shell gravity code which accurately reproduces the results of a full n-body code (Carlberg 2015b) for these very low mass clusters relative to the host galaxy. The shell code calculates self-gravity using only the gravitational monopole, that is, the gravitational acceleration at radius $r$ from the center of the cluster is $-G M_{c}(<r) /\left(r^{2}+\epsilon^{2}\right)$ in the radial direction, where $M_{c}(<r)$ is the cluster mass interior to the location of some particle and the softening, $\epsilon$, is set equal to one-fifth of the core radius. T he softening is set equal to 0.2 of the core radius of the King model, which varies with the scaling of the model to the tidal radius. Typically $\epsilon$ is about 1 pc.

Stellar mass loss is incorporated with an analytic model. Here, all particles have masses that decline as $m_{p}(t)=m_{p}(0)\left[1-\left(t / t_{w}\right)^{\gamma}\right.$. We use $t_{w}=16000(\simeq 560$ Gyr) and $\gamma=0.5$ which approximately describes the mass loss for ages beyond the evolution over 1-14 Gyr. At the final moment of the simulations the individual particles have lost $16 \%$ of their initial mass. This mass change makes little difference to the outcome.

A set of 24 star clusters is placed on circular orbits within the infalling satellite galaxy, 12 at the satellite's scale radius $a_{s}$ and 12 at $\frac{1}{2} a_{s}$. The clusters are in a disk that is tilted at $45^{\circ}$ with respect to the plane of the host galaxy potential. In physical terms, the clusters begin within about $10 \mathrm{kpc}$ of the center of their satellite host, which is itself at a distance of about $250 \mathrm{kpc}$ from the center of the main host galaxy.

Figure 1 shows a typical infall path of the satellite and a single cluster. The cluster orbits around the center of the infalling galaxy which is losing mass and spreading out as it falls in. When the satellite galaxy has fallen to a galactocentric radius of 5-10 units its mass has dropped to about half its initial value and the star clusters are unbound from the dissolving satellite galaxy. The satellite galaxy in Figure 1 has $M_{s}=0.3$. A satellite with $M_{s}=2$ makes only a one quarter turn orbit before plunging to the center.

\subsection{A Simple Model of Cluster Internal Dynamics}

The King model clusters are effectively collisionless over a model Hubble time. Hence, with only weak tidal heating, evaporation of star particles will be very slow, with even the most eccentric orbits losing less than $10 \%$ per Hubble time, as shown in Figure 2.

The internal dynamics of globular clusters is a rich subject which continues to develop with ever more realistic n-body simulations and sophisticated dynamical analysis. The dynamical evolution of star clusters is reviewed in (Spitzer 1987: Binney \& Tremaine 2008: Davies 2013). In brief, two body relaxation leads to the core shrinking and envelope expansion until heating from central binary and multiple star systems intervenes to limit core collapse and sometimes cause core oscillations. The gravitational collisions, largely in the central region, cause stars in the envelope to gradually move to larger orbits until they reach a tidal surface and become unbound from the cluster. Gieles et al. (2011) find that clusters with mass greater than $(8 \mathrm{kpc} / R) 4 \times 10^{4} \mathrm{M}_{\odot}$, are in the expansion dominated phase of evolution, where $R$ is the galactocentric radius of the cluster. The vast majority of thin Milky Way streams are located outside the solar circle and well beyond. A typical halo cluster at $15 \mathrm{kpc}$ that is more massive than $2 \times 10^{4} \mathrm{M}_{\odot}$ will be in the expansion phase of evolution, which simplifies the modeling requirements.

The controlling timescale for internal evolution of a cluster of mass half mass $M_{1 / 2}$ is the relaxation time at the half mass radius, $r_{1 / 2}$,

$$
t_{r h} \propto M_{1 / 2}^{1 / 2} r_{1 / 2}^{3 / 2} \propto \sqrt{\left\langle v^{2}\right\rangle} r_{1 / 2}^{2}
$$

(Spitzer 1969, 1987), where $\sqrt{\left\langle v^{2}\right\rangle}$ is a representative halt-mass velocity dispersion. The relaxation process causes the envelops to expand in a self-similar manner (Hénon 1961; Freitag et al. 2006a b).

The goal here is to understand how a somewhat more complete cluster and galaxy assembly history affects the 


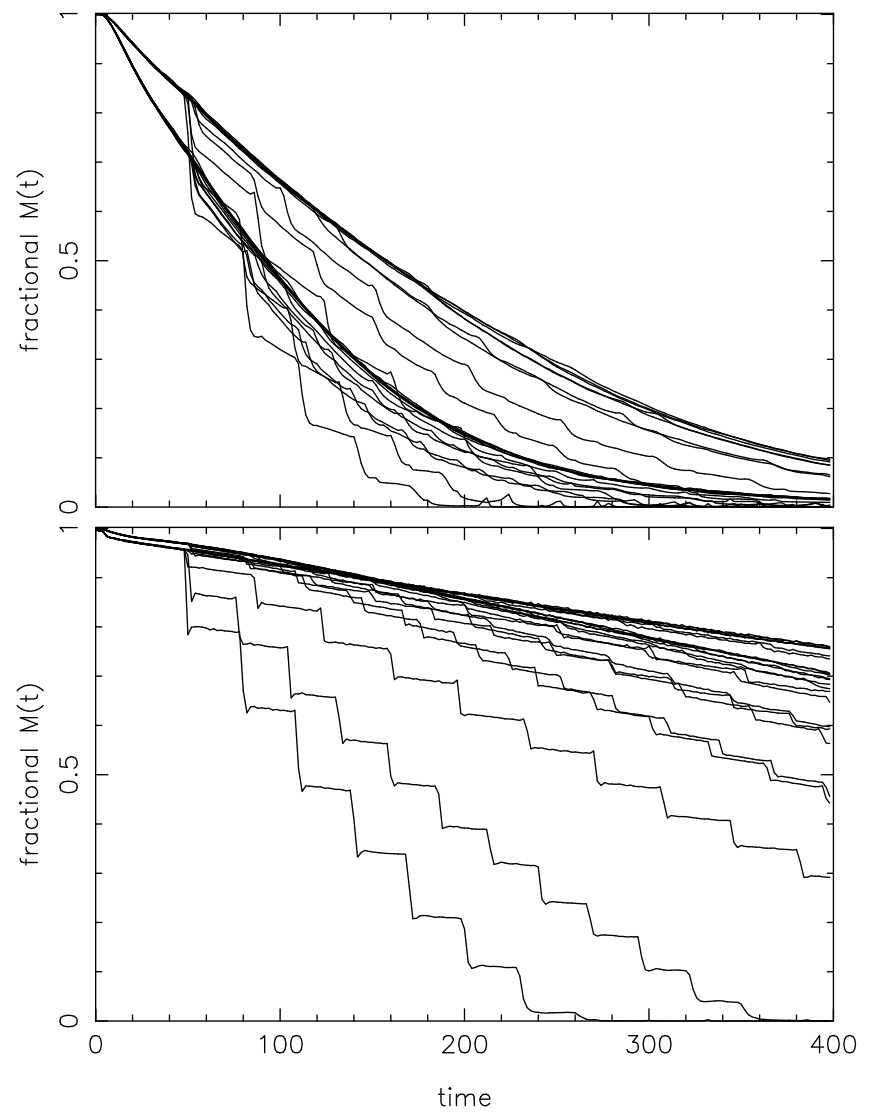

FIG. 3.- Mass as function of time for the 24 clusters each initially with mass $0.9 \times 10^{4} \mathrm{M}_{\odot}$ in the top panel and $0.9 \times 10^{5} \mathrm{M}_{\odot}$ in the bottom panel. The heating model is incorporated into all of the clusters, with the same parameters for all. Highly eccentric orbits have large mass loss variations around the orbit.

stream properties. The heating process is simply modeled with the addition of small velocity kicks to stars in the clusters. The required size of the velocity increments is estimated from the diffusion equation for stellar velocities (Binney \& Tremaine 2008) in a cluster with collisions,

$$
\frac{d\left\langle v^{2}\right\rangle}{d t}=\zeta \frac{\left\langle v^{2}\right\rangle}{t_{r h}\left(M_{1 / 2}, r_{1 / 2}\right)}
$$

where Gieles et al. (2011) find that $\zeta \simeq 0.1$. In a dynamically correct Monte Carlo simulation an energy increase to outer stars would be extracted from inner stars and binaries (Giersz 1998). This initial investigation does not require an accurate internal model of the cluster, so energy is simply added to the envelope at the predicted rate.

The velocity increments, $\delta v$, will be applied at discrete intervals, $\delta t_{r}$. Over some simulation time $T$, the velocity gains are $\Delta\left\langle v^{2}\right\rangle=T / \delta t(\delta v)^{2}$. Comparing Equations 3 and 4 gives the required step-wise velocity changes,

$$
(\delta v)^{2} \propto \zeta \frac{\delta t}{T} \frac{M_{1 / 2}^{1 / 2}}{r_{1 / 2}^{5 / 2}} .
$$

A practical implementation of Equation 5 is every $\delta t$ in time to add random velocities in all three directions
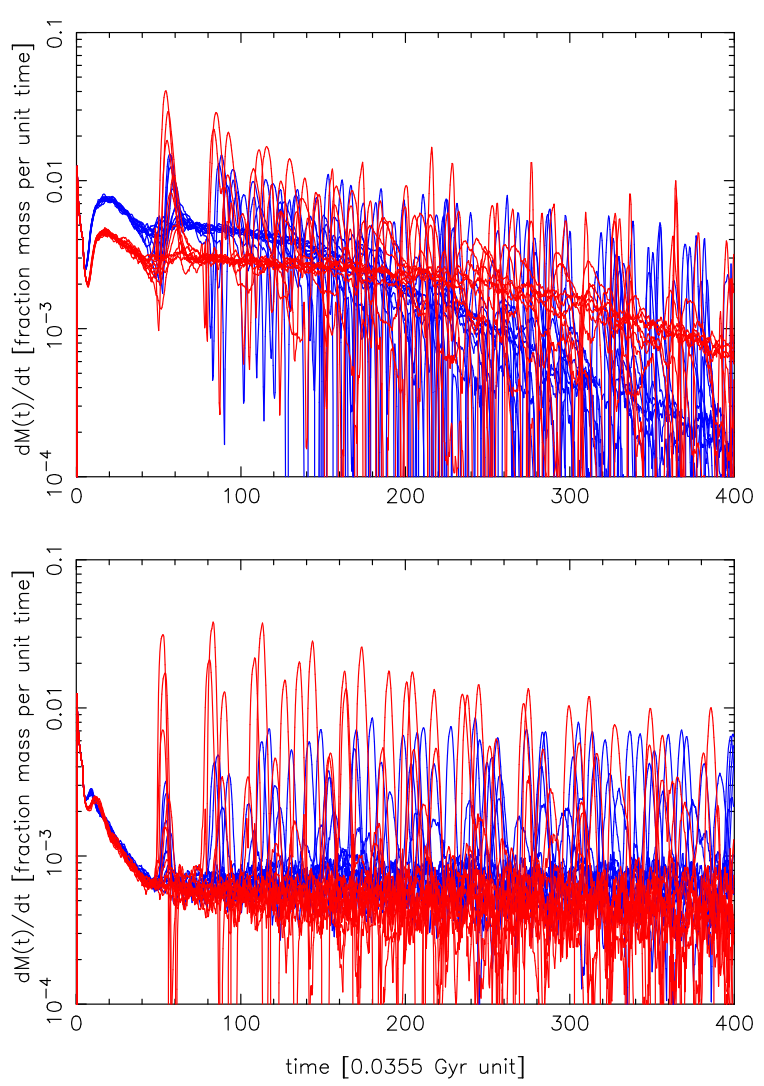

FIG. 4. - Mass loss rate, normalized to the initial mass, as function of time for the 24 clusters each with initial mass $0.9 \times 10^{4} \mathrm{M}_{\odot}$ in the top panel and $0.9 \times 10^{5} \mathrm{M}_{\odot}$ in the bottom panel. The inner 12 clusters use blue lines and the outer 12 are in red.

drawn from a Gaussian velocity distribution of width,

$$
\delta v=C \sigma\left(\frac{\delta t}{T}\right)^{1 / 2}\left(\frac{M_{1 / 2}}{10^{-7}}\right)^{1 / 4}\left(\frac{r_{1 / 2}}{10^{-3}}\right)^{-5 / 4},
$$

in the dimensionless units of the calculation, where $\mathrm{C}$ is a constant around unity that absorbs $\zeta$ and $\sigma$ is a characteristic velocity dispersion in the cluster. The $\delta v$ value is used to generate random velocity offsets in the $x y z$ directions from a Gaussian distribution. In this paper $\delta v$ is fixed for any model cluster, however, it could be normalized to the evolving RMS velocity dispersion of the cluster.

A little experimentation is required to fine tune the parameters controlling the heating process. An outer radius for the evaluation of the RMS velocity and the half mass radii of the clusters is selected to $r_{m}=0.02$ units $(160 \mathrm{pc})$ which is somewhat larger than the initial tidal radius of the clusters. No heating is done for particles beyond $r_{m}$ or inside the radius containing $1 / 3$ of the mass inside $r_{m}$, the latter to roughly replicate the results of detailed collisional n-body models. The heating calculation is done every $\delta t$ of 100 steps $\left(0.71 \times 10^{7}\right.$ yr). Equation 6 is evaluated with $C \sigma \sqrt{\delta t / T}$ set equal to $3 \times 10^{-4}$ for $T=400$, the duration of the simulation. The simulations have 200,000 time steps so this random walk process happens 2000 times. 


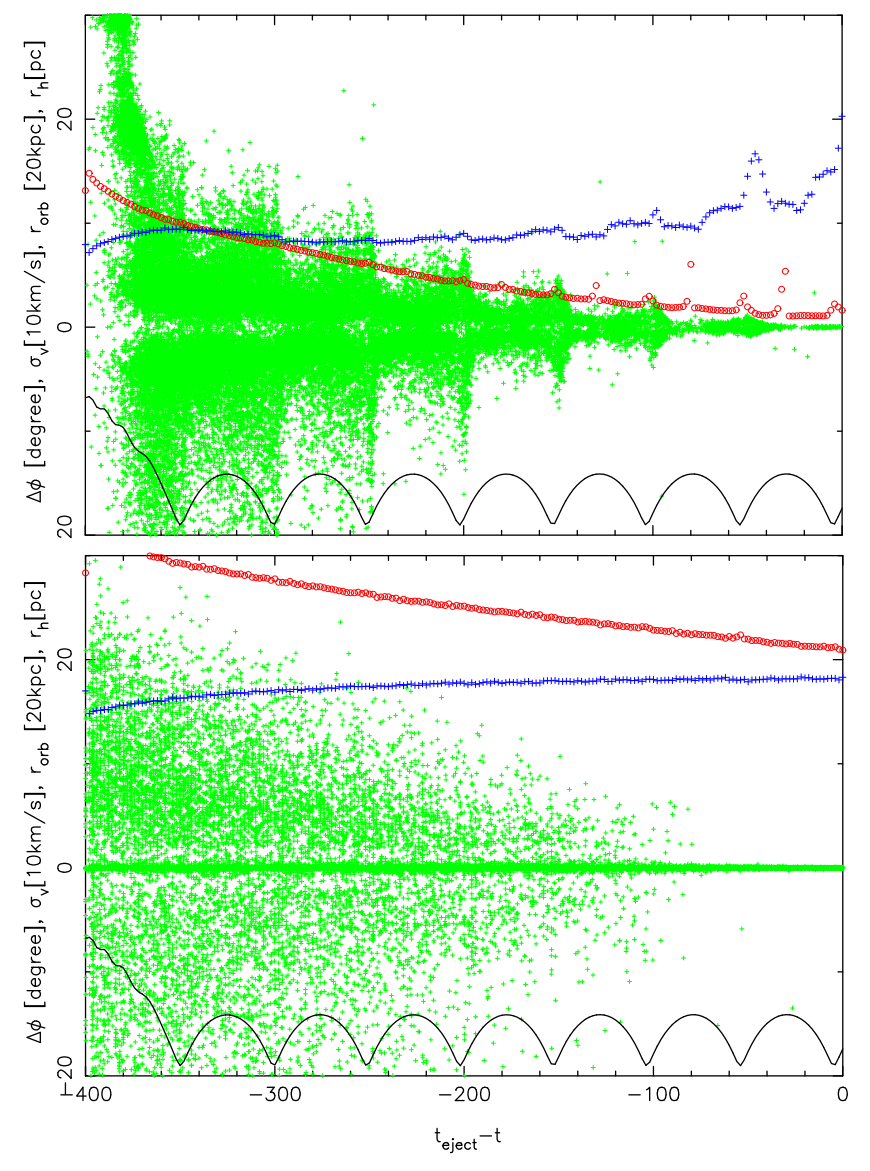

FIG. 5.- Evolution of the satellite and particles released into the stream with time for single clusters on the same orbit with mass $0.9 \times 10^{4}$ and $0.9 \times 10^{5} \mathrm{M} \odot$ in the top, middle and bottom panels, respectively. Red circles are the $3 \mathrm{D}$ velocity dispersion in $\mathrm{km} \mathrm{s}^{-1}$ times 10. Blue plus signs are the half mass radius of the remaining star cluster in parsecs. Green is the angular distance on the sky between the satellite center and the released particles, measured in degrees. The black line is the dimensionless orbital radius in $20 \mathrm{kpc}$ units, offset by -20 to the bottom of the plot. The orbital eccentricity is 0.86 .

\section{CLUSTER AND STREAM EVOLUTION}

The simulations have 24 star clusters arranged in two rings of 12 clusters in the satellite galaxy. Simulations with all clusters having an initial mass of $0.9 \times 10^{4} \mathrm{M}_{\odot}$ and $0.9 \times 10^{5} \mathrm{M}_{\odot}$ are done. The simulations reported use a satellite galaxy with $M_{s}=2$ which can be considered a $22 \%$ mass satellite, relative to the MW2014 model to its nominal virial radius. The simulations run for 400 time units, or 14.2 Gyr. All simulations use the same heating parameters and merger model. Simulations have been run for many other satellite masses, satellite starting conditions, sub-halo contents of the host galaxy and the cluster internal relaxation parameters, however the set presented provides a good guide to understanding the general outcomes.

Figure 3 shows the fractional cluster mass as a function of time for initial cluster masses of $\sim 10^{4} \mathrm{M}_{\odot}$ (top panel) and $\sim 10^{5} \mathrm{M}_{\odot}$ (bottom panel). As expected the $\sim 10^{4} \mathrm{M}_{\odot}$ clusters largely, but not completely, evaporate in a Hubble time whereas the $\sim 10^{5} \mathrm{M}_{\odot}$ clusters, on the average, lose less than half their mass. The mass loss rates are shown in Figure 4 with the clusters separated into the inner ring (blue) and the outer ring (red). The peaks in the mass loss rates with time occur at orbital apocenter, where stars that were unbound earlier move away from the cluster. The inner clusters initially have higher mass loss rates, most clearly shown in the upper panel of Figure 4, which is a consequence of the higher tidal fields nearer to the center of the satellite galaxy. The outer clusters tend to be launched into more eccentric orbits where they experience stronger tidal fields at orbital pericenter and have higher mass loss rates. Two of the 24 clusters of the bottom panel have sufficiently elliptical orbits that tidal fields help drive complete evaporation.

The $\sim 10^{5} \mathrm{M}_{\odot}$ clusters here are comparable to the direct n-body simulations for a similar mass cluster on a similar range of orbital eccentricities (Zonoozi et al. 2017). All the clusters have orbital apocenters near 250 kpc. The two clusters that dissolve have pericenters of 4 and $6 \mathrm{kpc}$, whereas the two that drop to about $1 / 3$ and $1 / 2$ of their initial particle count (the masses drop a little more due stellar ) have pericenters of 8 and $10 \mathrm{kpc}$, respectively.

The characteristic dynamical quantities of a single cluster and its stream are illustrated in Figure 5 as a function of time for individual clusters at masses $\sim 10^{4}$ and $\sim 10^{5} \mathrm{M}_{\odot}$, in the top and bottom panels, respectively. The two clusters are on essentially the same orbit, with eccentricity 0.86 . The half mass radius of the clusters, around $20 \mathrm{pc}$, is well inside the tidal radius of the clusters, which varies around the orbit and is typically about 100 pc. The particle density distributions have no crisp cutoff. The half mass radius expands modestly, approximately $50 \%$, over the course of the cluster evolution as long as the cluster retains significant mass. The RMS velocity dispersion of the cluster stars is largely dependent on the cluster mass, dropping to zero if the cluster evaporates completely. The spray of (green) points in Figure 5 shows the current angular distance from the progenitor cluster (or its remnant center) of stream particles as a function of the time at which the star particle went through a fixed radius near the tidal surface. The decline of velocity dispersion as the cluster evaporates will be directly reflected in the properties along the stream. The lower mass cluster loses relatively more stars when the cluster was still in the satellite galaxy. These stars spread to relatively large angular distances away from the from the progenitor cluster. The particles that evaporate late do so at such low velocity dispersions that they remain relatively close to the cluster compared to the higher drift velocities of particles released earlier. The more massive clusters lose relatively few stars while orbiting in the satellite galaxy, after which the cluster maintains a fairly uniform low rate of mass loss ejecting stars at velocities that only allows particles to reach angular separations of $20^{\circ}$ or so from the cluster. Stream properties are cluster mass dependent.

The mean particle density summed over all 24 streams from a set of equal mass clusters is plotted as function of azimuthal angle with the orbit projected onto the $\mathrm{x}$ y plane in Figure 6. The average largely removes the orbital phase variations of individual streams. The particle density in the lower mass streams is higher because the clusters essentially completely evaporate. The cluster models all have 50,000 star particles, so a conversion 


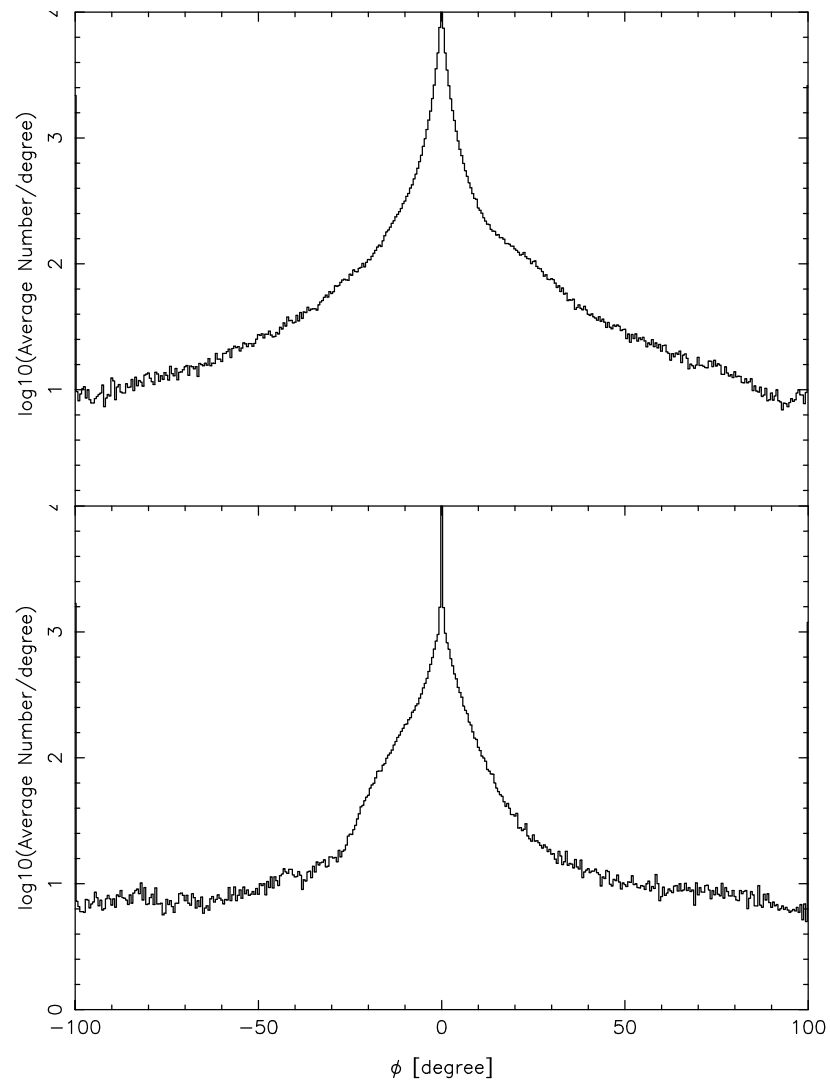

FIG. 6.- The average particle density along the stream projected onto the $\mathrm{x}-\mathrm{y}$ plane averaging together the 24 clusters with mass $\sim 10^{4} \mathrm{M}_{\odot}$ in the top panel and $\sim 10^{5} \mathrm{M}_{\odot}$ in the bottom panel, both at time 400 (14 Gyr). A conversion to stellar density would boost the relative density of the lower panel a factor of ten.

to stellar density the actual stellar density would lead to a relative increase of a factor of ten for the higher initial mass cluster streams of the bottom panel. That is, the higher mass clusters here will, on the average, be easier to find on the sky, for the same orbital parameters. Yet higher mass clusters evaporate so slowly that their streams are very low density. An implication is that star clusters with initial masses around $\sim 10^{5} \mathrm{M}_{\odot}$ will produce the highest sky density star streams.

The density along individual streams from the inner dozen clusters at $\sim 10^{4}$ and $\sim 10^{5} \mathrm{M}_{\odot}$ clusters done in a simulation with sub-halos present is shown in Figure 7. There is a substantial variation in stream density profiles depending on the details of the orbits and the sub-halo interactions. The considerable variation of the stream density profiles from one orbit to the next is clear readily visible, much of it due to orbital phase where the stream near apocenter increases in linear density on the sky and declines near pericenter. Sub-halo induced gaps are visible in most of the stream densities. The orbits of both clusters and sub-halos are the same for the two sets of cluster masses the gaps are nearly identical as are the sub-halo interactions, so the differences between the two mass sets can largely be attributed to cluster mass dependence. The most prominent gaps range in size from a degree up to about ten degrees, with the most visible gaps being around 5 degrees in length.

The $\mathrm{x}-\mathrm{y}$ projections of streams from $\sim 10^{4} \mathrm{M}_{\odot}$ and

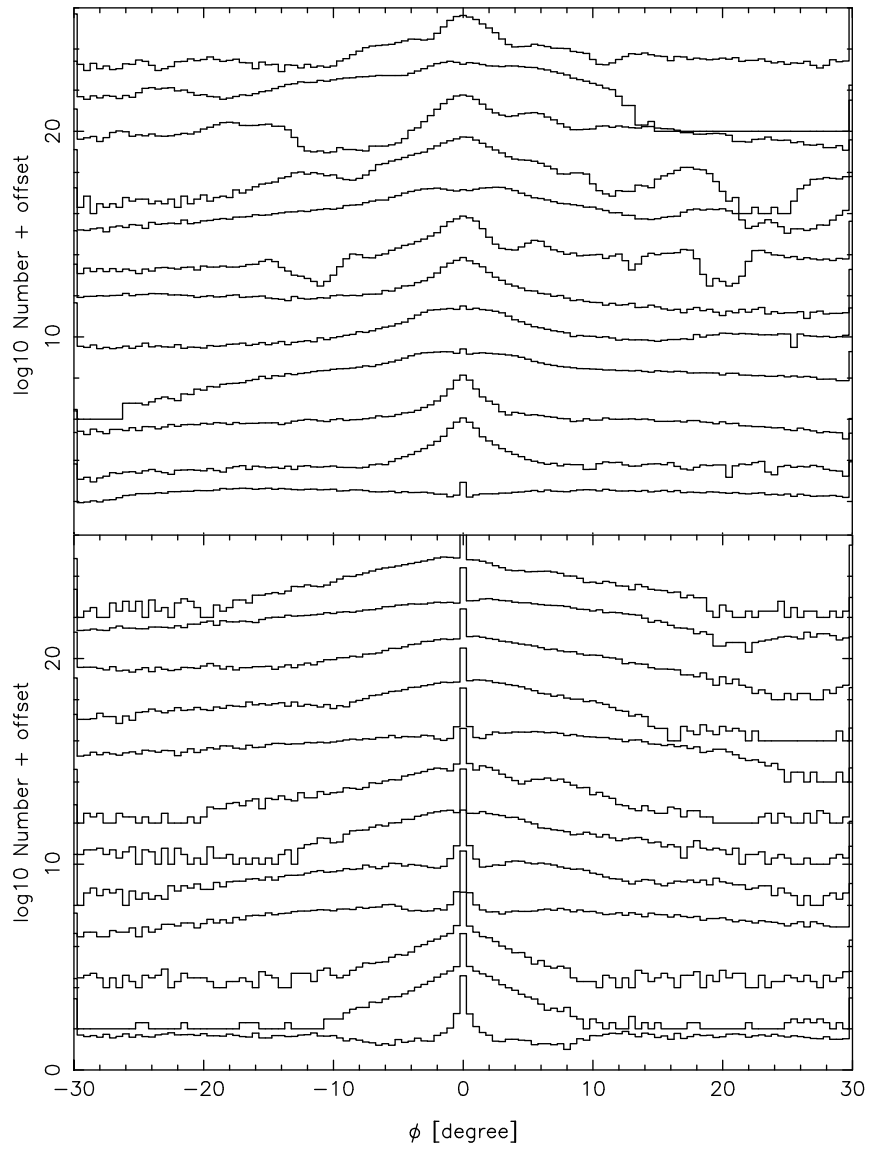

FIG. 7.- The density along the stream for individual streams from the 12 inner clusters with masses $0.9 \times 10^{4}$ and $0.9 \times 10^{5} \mathrm{M}_{\odot}$ in the top and bottom panels, respectively. Individual streams vary as a result of orbital phase which increases density at apocenter and decreases it at pericenter. The $5^{\circ}$ scale density variations along an individual stream are the result of dark matter sub-halos passing through the streams.

$\sim 10^{5} \mathrm{M}_{\odot}$ clusters are shown in Figure 8. To a good approximation the cluster orbits are independent of the cluster masses, so for every cluster in the top panel there is a matching one at 10 times the initial mass in the bottom panel. All of these clusters are on orbits with apocenters near $240 \mathrm{kpc}$ but with a wide range of pericenters. The lower mass clusters on the top make narrower streams and the sky density is less concentrated to the location of progenitor cluster, as also shown in Figure 6 The satellite galaxy model potential can be adjusted to produce smaller apocenters.

\section{VISIBILITY OF STREAMS ON THE SKY}

The simulations here lead to the conclusion that streams from star clusters at masses around $10^{5} \mathrm{M}_{\odot}$ should be the most visible on the sky, when converted to number of stars per unit angular length of stream. The density and width varies along the stream, with the ends being relatively low linear density and relatively wide, hence reduced surface brightness relative to near the progenitor cluster.

Streams are very low sky density features which in photometric data alone become detectable through the use of color-magnitude diagram filtering (Rockosi et al. 2002 which optimally weights some desired common age, 


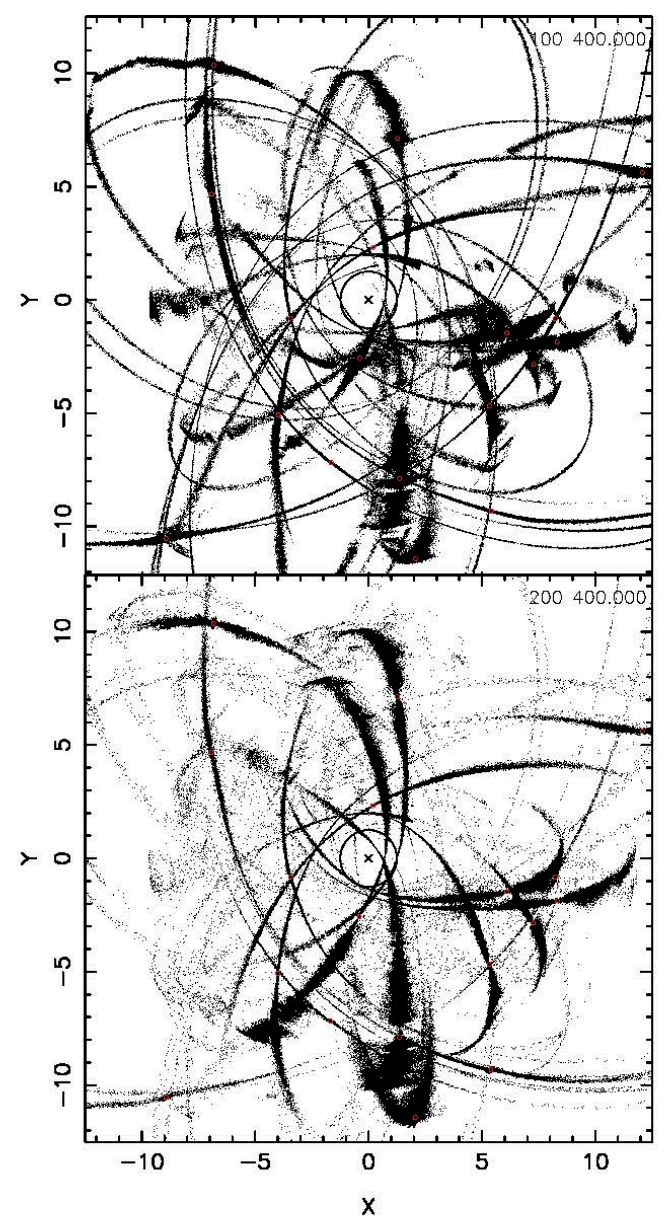

FIG. 8. - The tidal tails of a set of 24 infalling clusters each with mass $\sim 10^{4} \mathrm{M}_{\odot}$ (top) and $\sim 10^{5} \mathrm{M}_{\odot}$ bottom. The grid scale is in units of $8 \mathrm{kpc}$ with a circle of radius $10 \mathrm{kpc}$ around the center. The red circles are the locations of the nominal cluster centers, some of which have no remnant cluster.

metallicity and distance population of stars in preference to field stars. Even with such procedures currently available data generally lead to streams with a signal-to-noise per degree length of stream of a few. If the stream is at a sufficiently large distance such that the large number of stars near the turnoff from the main sequence are not within the sample, about 15-20 kpc for current images, then the signal to noise drops further. The outcome is that finding star streams is difficult at best and essentially impossible with current data to detect lower density regions of streams.

In Figure 9 the sky density is shown for all 24 streams of the bottom panel of Figure 8 . In the upper panel of Figure 9 any pixel on the sky with a star is black, irrespective of the numbers. A pixel is 0.002 units in a the Aitoff-Hammer coordinate which has a maximum of $2 \sqrt{2}$. Scaling to $360^{\circ}$ indicates that the pixels are approximately $0.13^{\circ}$ on a side. To more accurately represent sky density, in the middle panel the pixel density is deweighted with the inverse distance squared for stars beyond $16 \mathrm{kpc}$ and the resulting pixel density plotted with a grey scale from 0 to 4 units. The bottom panel shows the expected sky densities for the $\sim 10^{4} \mathrm{M}_{\odot}$ streams on

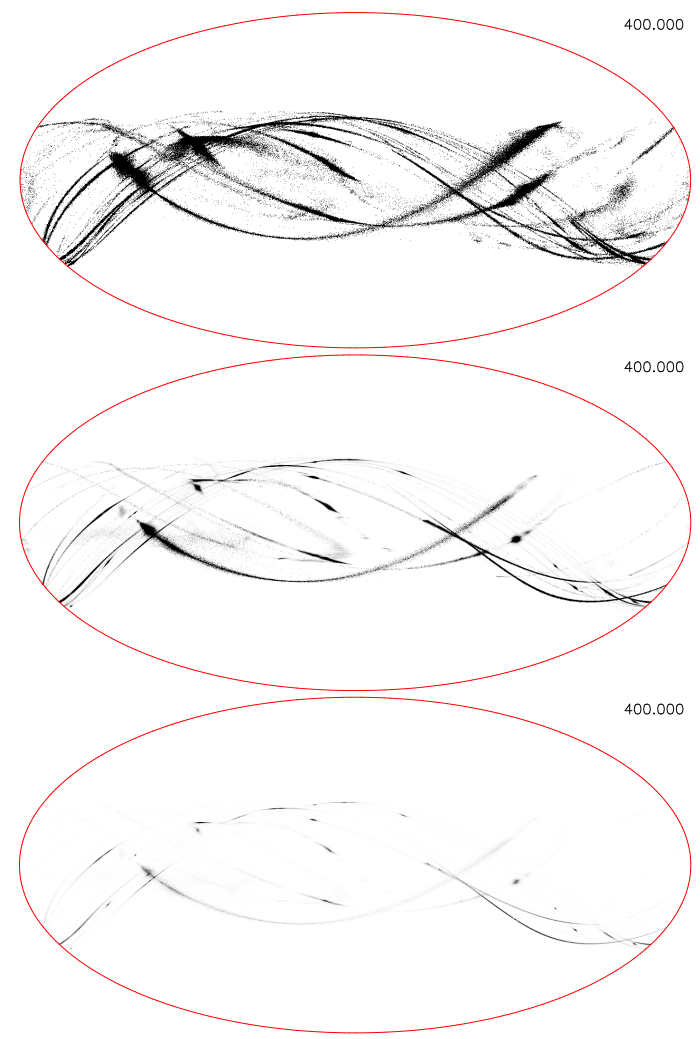

FIG. 9.- The Aitoff-Hammer projection of the 24 clusters initiated with masses of $0.9 \times 10^{5} \mathrm{M}_{\odot}$ (top two panels) and $0.9 \times 10^{4} \mathrm{M}_{\odot}$ (bottom panel) in the $M_{s}=2$ infall model. In the top panel any pixel with a particle in it is black, a $0-1$ grey scale. All particles are equally visible in the top panel. In the middle and bottom panels particles beyond $16 \mathrm{kpc}$ are weighted by their inverse distance squared. In the middle panel the grey scale is $0-4$ to emphasize the higher density regions, and in the bottom the grey scale is 040 which puts the middle and bottom panel on the same stellar brightness scale.

a grey scale of 0 to 40 to mimic the conversion to the brightness of stars.

The weighted map of the sky density of streams is a much more realistic view of what should be expected in current sky maps (Ngan et al. 2015). The streams are thinner and shorter than they would be if all stars were detected, which will eventually be possible. However, the streams do not disappear entirely. That is, even though the length and width of streams is likely being underestimated, the counts of streams is likely to be accurate for streams within double the color-magnitude main sequence turn-off detection distance. The details of the stream selection function will be studied more quantitatively in a future paper.

\section{STREAM - CLUSTER COMPLEMENTARITY AND GALAXY ASSEMBLY}

For every completely dissolved globular cluster, there will be a thin star stream with its stars distributed close to the orbit of the cluster. The streams also provide an indicator of when their progenitor cluster fell in through their length. A cluster recently merged into the galaxy will have a short tail and old additions should have very long tails. Creating a sample of both streams and glob- 


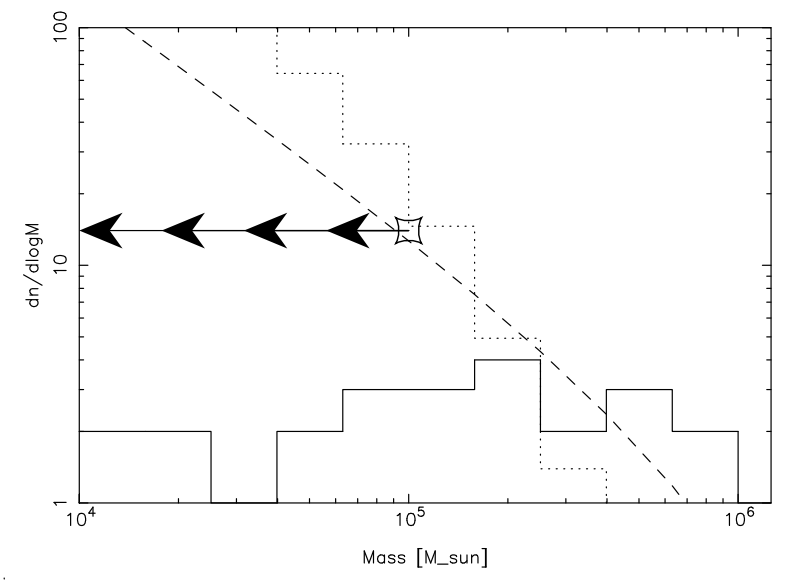

FIG. 10.- The number of globular clusters as a function of mass in the PS1-3 $\pi$ survey (solid histogram) compared to the number of dense stellar systems expected to be formed in an $M^{-2} \exp \left(-M / M_{*}\right)$ distribution normalized to the top half dozen clusters (dashed line). The dotted histogram is the cumulative sum of the difference between the assumed initial distribution and the current numbers, which are expected to become stellar streams. The pincushion symbol and left arrows is for the 14 streams recovered in the PS1-3 $\pi$ area. The few stream masses known are in the few $\times 10^{4} \mathrm{M} \odot$ range. The streams are put at $10^{5} \mathrm{M} \odot$ primarily on the basis that the heaviest "lost" clusters should be the most visible star streams so the stream mass should be as high as allowed.

ular clusters with well-understood sampling is not yet possible, but an interesting start can be made.

The PS1-3 $\pi$ survey provides nearly uniform sky coverage over a large sky area north of Declination -30. The Bernard et al. (2016) stream search is defined within a maximum distance of of $35 \mathrm{kpc}$, which here is taken as a galactocentric radius. The minimum galactocentric radius is $10 \mathrm{Kpc}$, inside of which streams are not found, possibly because they encounter the stellar disk and its massive gas clouds and are quickly eroded (Amorisco et al. 2016). Within the PS1-3 $\pi$ sky Bernard et al. (2016) recover 9 previously known thin streams (wider, dwarf galaxy streams are excluded here), and, find 5 new ones for a total of 14. A few more streams are known in this sky area from other work (compiled in Grillmair \& Carlin (2016)), but PS1-3 $\pi$ provides the most uniform and large area of sky available at the moment. A few of the streams have mass or luminosity estimates, such as GD1 estimated to have a mass $2 \times 10^{4} \mathrm{M}_{\odot}$ (Koposov et al. 2010). There is no estimate of how much mass associated with any stream may be missed at low surface brightness or or dust obscuration. The simulations presented here indicate that the streams are likely in the mass range around $10^{5} \mathrm{M}_{\odot}$. Streams above this mass limit should be readily visible.

The Harris (1996) catalog is used to construct a sample of globular clusters in the same sky volume. Remnant halo clusters are those visible within the same sky volume as the star streams. To a first approximation, the obscuration of the galactic disk should be about the same for streams and the halo population of globular clusters. An upper limit on metallicity of $[\mathrm{Fe} / \mathrm{H}]$ of -1 is imposed to focus on halo clusters, although this only eliminates 3 very low luminosity clusters out of 27 in the PS1-3 $\pi$ volume. The selected clusters have a mean galactocentric distance of $19 \mathrm{kpc}$. Masses are assigned to the globular clusters using a uniform M/L of 2 solar units and plotted in Figure 10. The pin cushion symbol shows the 14 PS1 streams, assigned a mass of $10^{5} \mathrm{M}_{\odot}$, although that is likely an upper-limit, as indicated with the arrows.

The mass distribution of current epoch dense star clusters is a steep power law, approximately $M^{-2}$ (Portegies Zwart et al. 2010). Since the high mass clusters in the halo are expected to have largely retained their initial mass, the $M^{-2}$ can be fit to the higher mass clusters to predict the numbers at lower masses. In Figure 10 the dashed line is $7\left(M / M_{*}\right)^{-1} \exp \left(-M / M_{*}\right) \Delta M / M$ with $M_{*}=10^{6} \mathrm{M}_{\odot}$ and $\Delta \log _{10} M=0.2$, which approximately fits the mass distribution of the half dozen clusters above $3 \times 10^{5} \mathrm{M}_{\odot}$. The dotted histogram is the cumulative difference between the clusters observed to be present and their assumed progenitor numbers, that is, the number of "lost" clusters. The difference between the 28.6 clusters expected from the dense star cluster progenitor distribution and the 14 clusters more massive than $10^{5} \mathrm{M}_{\odot}$ is 14.6. Given the uncertainty on the stream masses this primarily means that the progenitor cluster distribution could not have been steeper than $M^{-2}$. It also suggests that essentially all of the massive streams in the PS1 volume have been found, but there could be many more lower mass streams.

\section{DISCUSSION}

The processes of galaxy assembly and star cluster evaporation need to be included to provide an accurate dynamical history of the development of stellar streams. This paper develops a toy model with a simple merger model and a cluster heating function that approximates the internal dynamics of star clusters. Clusters expand and cool as they lose mass, causing the stream linear density to vary with distance from the progenitor clusters. The density decline of streams in an evolving potential contrasts with the effectively constant linear density of streams produced in static background potentials (Küpper et al. 2012, Bovy 2014; Carlberg 2015a), setting aside orbital phase variation. The portions of the tidal streams created prior to merging of the satellite galaxy into the host are often very widely dispersed.

The heated model clusters have mass loss in rough agreement with detailed cluster dynamical studies. The clusters having a mass around $10^{5} \mathrm{M}_{\odot}$ lose about one third of their mass in a Hubble time and $10^{4} \mathrm{M}_{\odot}$ clusters largely evaporate in about $2 / 3$ of a Hubble time. The linear density of the resulting stellar streams declines about a factor of ten between $10^{\circ}$ and $50^{\circ}$ from the progenitor cluster, although there is considerable case to case variation. When projected onto the sky, the decline of the mean density away from the progenitor helps explain why the readily visible parts of most streams are relatively short, even though the simulated streams often wrap a good fraction of their orbit around the galaxy. Dark matter sub-halos create gaps along the streams, but because gaps are largely changes parallel to the orbital motion (Yoon, Johnston \& Hogg 2011), the streams remains robust structures (Helmi \& White 1999) and not readily blurred out. The result of these models is an improved 
conceptual understanding of the development of streams and their sky density within an accreting halo, but almost every aspect of the simulation can be improved in more detailed future investigations.

The globular clusters around $10^{5} \mathrm{M}_{\odot}$ that dissolve should all produce streams sufficiently bright to be detectable with optimized color-magnitude filtering methods if they are in an accessible part of the sky. Therefore streams when combined with the remnant globular clusters in the same volume can provide an estimate of the total cluster population present at early epochs. The current data do not have well determined stream masses and the stream selection function is not well understood. However, an interesting star can be made with the PS1$3 \pi$ survey which finds 14 streams (Bernard et al. 2016) in its volume. In the same sky volume there are 14 globular clusters to $10^{5} \mathrm{M}_{\odot}$. Making the assumption that all the streams are all at or above $10^{5} \mathrm{M}_{\odot}$ in total mass then leads to the conclusion that there were 28 clusters in in the progenitor cluster population to $10^{5} \mathrm{M}_{\odot}$, which would be in accord with the expectations of an $M^{-2}$ initial cluster mass distribution. The minimum mass of the current stream sample is almost certainly somewhat lower than $10^{5} \mathrm{M}_{\odot}$ which then makes $M^{-2}$ the upper limit to the shape of the progenitor mass distribution. A possibly interesting implication is that if binary black holes (BBH) at least partially form in clusters with low metallicity masses around $10^{5} \mathrm{M}_{\odot}$ (Chatterjee et al. 2017) with the first few Gigayears prior to assembly into the Milky Way, then a prediction of BBH numbers based on the current globular cluster population is an underestimate, by a factor of two or less, based on the stream counts.

This research was supported by CIFAR and NSERC Canada. An anonymous referee provided constructive criticism and pointed to an error in the mass scaling in an earlier version.

\section{REFERENCES}

Amorisco, N. C., Gómez, F. A., Vegetti, S., \& White, S. D. M. 2016, MNRAS, 463, L17

Bernard, E. J., Ferguson, A. M. N., Schlafly, E. F., et al. 2016, MNRAS,

Binney, J. 2008, MNRAS, 386, L47

Binney, J., \& Tremaine, S. 2008, Galactic Dynamics: Second Edition, Princeton University Press

Bournaud, F., Duc, P.-A., \& Emsellem, E. 2008, MNRAS, 389, L8

Bovy, J. 2014, ApJ, 795, 95

Bovy, J. 2015, ApJS, 216, 29

Bovy, J., Erkal, D., \& Sanders, J. L. 2017, MNRAS, 466, 628

Bovy, J., Bahmanyar, A., Fritz, T. K., \& Kallivayalil, N. 2016, ApJ, 833, 31

Boylan-Kolchin, M., Ma, C.-P., \& Quataert, E. 2008, MNRAS, 383, 93

Carlberg, R. G. 2012, ApJ, 748, 20

Carlberg, R. G. 2015a, ApJ, 800, 133

Carlberg, R. G. 2015b, ApJ, 808, 15

Carlberg, R., \& Grillmair, C. 2016, ApJ, 830, 135

Chatterjee, S., Rodriguez, C. L., \& Rasio, F. A. 2017, ApJ, 834, 68

Davies, M. B. 2013, Planets, Stars and Stellar Systems. Volume 5 : Galactic Structure and Stellar Populations, 5, 879 ed Oswalt, Terry D.; Gilmore, Gerard (ISBN 978-94-007-5611-3. Springer Science+Business Media Dordrecht)

Eyre, A., \& Binney, J. 2011, MNRAS, 413, 1852

Fall, S. M., \& Rees, M. J. 1977, MNRAS, 181, 37P

Fall, S. M., \& Rees, M. J. 1985, ApJ, 298, 18

Fall, S. M., \& Zhang, Q. 2001, ApJ, 561, 751

Freitag, M., Gürkan, M. A., \& Rasio, F. A. 2006, MNRAS, 368, 141

Freitag, M., Rasio, F. A., \& Baumgardt, H. 2006, MNRAS, 368, 121

Gieles, M., Heggie, D. C. \& Zhao, H. 2011, MNRAS, 413, 2509

Giersz, M. 1998, MNRAS, 298, 1239

Gnedin, O. Y., \& Ostriker, J. P. 1997, ApJ, 474, 223

Grillmair, C. J., \& Carlin, J. L. 2016, Astrophysics and Space Science Library, 420, 87

Grillmair, C. J., \& Dionatos, O. 2006a, ApJ, 643, L17

Grillmair, C. J., \& Dionatos, O. 2006b, ApJ, 641, L37
Harris, W. E. 1996, AJ, 112, 1487

Helmi, A., \& White, S. D. M. 1999, MNRAS, 307, 495

Hénon, M. 1961, Annales d'Astrophysique, 24, 369

Hernquist, L. 1990, ApJ, 356, 359

Ibata, R. A., Lewis, G. F., Irwin, M. J., \& Quinn, T. 2002, MNRAS, 332, 915

Johnston, K. V., Spergel, D. N., \& Haydn, C. 2002, ApJ, 570, 656

Klypin, A., Kravtsov, A. V., Valenzuela, O., \& Prada, F. 1999, ApJ, 522, 82

Koposov, S. E., Rix, H.-W., \& Hogg, D. W. 2010, ApJ, 712, 260

Kravtsov, A. V., \& Gnedin, O. Y. 2005, ApJ, 623, 650

Küpper, A. H. W., Lane, R. R., \& Heggie, D. C. 2012, MNRAS, 420,2700

Lamers, H. J. G. L. M., Baumgardt, H., \& Gieles, M. 2013, MNRAS, 433,1378

Law, D. R., \& Majewski, S. R. 2010, ApJ, 714, 229

Moore B., Ghigna S., Governato F., et al., 1999a, ApJL, 524, L19

Navarro, J. F., Frenk, C. S., \& White, S. D. M. 1997, ApJ, 490, 493

Ngan, W., Bozek, B., Carlberg, R. G., et al. 2015, ApJ, 803, 75

Odenkirchen, M., Grebel, E. K., Rockosi, C. M., et al. 2001, ApJ, 548, L165

Portegies Zwart, S. F., McMillan, S. L. W., \& Gieles, M. 2010, ARA\&A, 48, 431

Renaud, F., Gieles, M., \& Boily, C. M. 2011, MNRAS, 418, 759

Renaud, F., Agertz, O., \& Gieles, M. 2016, arXiv:1610.03101

Rockosi, C.' M., Odenkirchen, M., Grebel, E. K., et al. 2002, AJ, 124,349

Sanders, J. L., \& Binney, J. 2013, MNRAS, 433, 1826

Sanders, J. L., Bovy, J., \& Erkal, D. 2016, MNRAS, 457, 3817

Spitzer, L., Jr. 1969, ApJ, 158, L139

Spitzer, L. 1987, Dynamical evolution of globular clusters, Princeton, NJ, Princeton University Press, 1987, 191 p.,

Yoon, J. H., Johnston, K. V., \& Hogg, D. W. 2011, ApJ, 731, 58

Whitmore, B. C., Zhang, Q., Leitherer, C., et al. 1999, AJ, 118, 1551

Zonoozi, A. H., Haghi, H., Kroupa, P., Küpper, A. H. W., \&

Baumgardt, H. 2017, MNRAS, 